\title{
Uso de volumetría y carga lesional en el seguimiento de pacientes con esclerosis múltiple. Experiencia local y revisión de la literatura
}

\author{
Dres. Jorge Cordovez $M^{(1)}$, Marcelo Gálvez $M^{(1)}$, Gonzalo Rojas $C^{(2)}$, Cristóbal Bravo C. ${ }^{(3)}$, Alejandro Cerda $E^{(4)}$.
}

1. Neurorradiólogo, Departamento de Diagnóstico por Imágenes, Clínica Las Condes, Santiago, Chile.

2. Ingeniero informático, Laboratorio de Imágenes Médicas, Clínica Las Condes.

3. Radiólogo, Departamento de Diagnóstico por Imágenes, Clínica Las Condes, Santiago, Chile.

4. Tecnólogo Médico, Departamento de Diagnóstico por Imágenes, Clínica Las Condes, Santiago, Chile.

\section{Use of lesion volumes and loads for monitoring patients with multiple sclerosis. Local experience} and literature review

\begin{abstract}
Multiple sclerosis (MS) is a common demyelinating disease that involves the central nervous system causing focal lesions in the brain and spinal cord causing diverse neurological development deficits, many of them severe and irreversible, affecting and invaliding a large percentage of young productive-aged patients. MRI exams have improved diagnostic capability compared to computed tomography, but in this decade the development of new magnets, coils and software have allowed the development of quantitative neuroradiology which achieves the evaluation of parameters such as total brain volume, of each of its structures, as well as semi-automated or automated counting of the lesion load, allowing better monitoring of each individual patient in relation to a particular event such as a new neurological deficit in an outbreak of the disease, a previously undetected cognitive impairment or in related to a given treatment. We will show our local experience using the FreeSurfer software in our habitual practice, as well as other post-processing software, this being the first experience of its use in multiple sclerosis published in our country.

Keywords: Brain volume, FreeSurfer, Magnetic resonance imaging, MIPAV software, Multiple sclerosis, Lession load,
\end{abstract}

Resumen. La esclerosis múltiple (EM) es una frecuente enfermedad desmielinizante que afecta el sistema nervioso central produciendo lesiones focales a nivel cerebral y medular que condicionan diversos déficit neurológicos evolutivos, muchos de ellos severos e irreversibles, afectando e invalidando un gran porcentaje de pacientes jóvenes en edad productiva. El estudio con resonancia magnética mejoró la capacidad diagnóstica respecto a la tomografía computada, pero en esta década el desarrollo de nuevos magnetos, bobinas y software han permitido desarrollar la neurorradiología cuantitativa que logra evaluar parámetros como el volumen cerebral global, de cada una de sus estructuras así como el recuento semi o automatizado de la carga lesional, lo que permite el mejor seguimiento de cada paciente individual en relación a un evento determinado como un nuevo déficit neurológico dentro de un brote de la enfermedad, un deterioro cognitivo no detectado previamente o en relación a un tratamiento determinado. Mostraremos nuestra experiencia local utilizando el software freesurfer en nuestra práctica habitual, así como otro software de postproceso, siendo la primera experiencia de su uso en esclerosis múltiple publicada en nuestro país.

Palabras clave: Carga lesional, Esclerosis múltiple, Freesurfer, MIPAV, Resonancia magnética, Volumen cerebral.

Cordovez J. Uso de volumetría y carga lesional en el seguimiento de pacientes con esclerosis múltiple. Experiencia local y revisión de la literatura. Rev Radiol 2013; 19(4): 156-164.

Correspondencia: Dr. Jorge Cordovez M. / jcordovezm@gmail.com

Trabajo recibido el 30 de agosto de 2013. Aceptado el 18 de diciembre de 2013.

\section{Introducción}

La esclerosis múltiple (EM) es una enfermedad desmielinizante frecuente que afecta el sistema nervioso central, produciendo lesiones focales que condicionan diversos déficit neurológicos evolutivos, muchos de ellos severos e irreversibles. Un importante porcentaje afecta a la población joven (entre los 20 y 40 años), más frecuente en mujeres (2:1), lo cual condiciona un importante impacto económico y social. No se ha definido exactamente su etiología lo que ha dificultado encontrar un tratamiento curativo. Por estos motivos, su estudio es una importante fuente 
de investigación en múltiples centros a nivel mundial.

Desde el punto de vista diagnóstico, uno de los avances importantes fue la incorporación de la resonancia magnética en la década de los '80, la cual nos permite visualizar las lesiones que eran omitidas por la tomografía computada. La incorporación de mejores magnetos, nuevas bobinas y software de postproceso ha aumentado la sensibilidad en el diagnóstico de esta enfermedad.

Una importante característica de la EM es la heterogeneidad en su expresión clínica(1) lo que se correlaciona con su heterogeneidad neuropatológica.

Diferentes cursos clínicos de la EM: forma recurrente-remitente $(R R)$, secundaria progresiva $(S P)$, primaria progresiva (PP), y la progresiva recidivante $(\mathrm{PR})^{(2)}$.

La EMRR se caracteriza por disfunciones neurológicas agudas autolimitadas seguida por un grado variable de recuperación. Por el contrario, EMPP se caracteriza por una disminución constante en la función neurológica desde el inicio, sin brotes evidenciables en la evolución clínica de la enfermedad. Aproximadamente 50 a $80 \%$ de los individuos que presentan un síndrome clínicamente aislado ya tienen lesiones en la RM, las que estarían con anterioridad (ocultas) a la actividad de la enfermedad ${ }^{(2)}$. Lucchinetti( ${ }^{(3)}$ ha propuesto cuatro distintas formas patológicas de la enfermedad donde existen lesiones multifocales con destrucción y reparación de la mielina, pérdida axonal y astrogliosis reactiva.

Para el diagnóstico clínico de rutina se utilizan secuencias potenciadas en T2 (Figura 1) que permiten al radiólogo evaluar la cantidad, posición y forma de las lesiones en la médula espinal y cerebro para establecer un diagnóstico presuntivo de esclerosis múltiple. En las secuencias T1 se visualizan los llamados "hoyos negros", que traduce un gran daño tisular.

En el año 2001 se incorporan los criterios de McDonald, los cuales establecen los criterios diagnósticos específicos para esclerosis múltiple en resonancia magnética en su diseminación en tiempo tanto como en espacio ${ }^{(4)}$.

Se ha observado una correlación entre los hallazgos en la RM y la actividad inflamatoria, la cual se ha utilizado como un biomarcador de progresión, con una buena correlación a los hallazgos neuropatológicos. Diversos estudios han confirmado la relación entre la captación de gadolinio ( $\mathrm{Gd}$ ) de las lesiones en T1 con actividad inflamatoria ${ }^{(5-8)}$. Esto ha llevado a la aceptación de la RM como un marcador sustituto en los diversos ensayos clínicos de tratamientos antinflamatorios para la EM.

Los avances técnicos en resonancia con magnetos de alto campo y nuevas bobinas, nos permiten la adquisición rutinaria de estudios volumétricos potenciados en T1 y T2. Con ello, y las nuevas técnicas automáticas o semiautomáticas de postproceso, nos permiten evaluar el volumen cerebral total, así como de diferentes regiones o estructuras cerebrales y determinar la carga lesional en cada uno de nuestros pacientes. Esto último es un importante elemento de juicio útil para determinar la evolución de la enfermedad en plazos breves tales como un año, lo cual nos introduce en la utilización de parámetros cuantitativos en esclerosis múltiple.
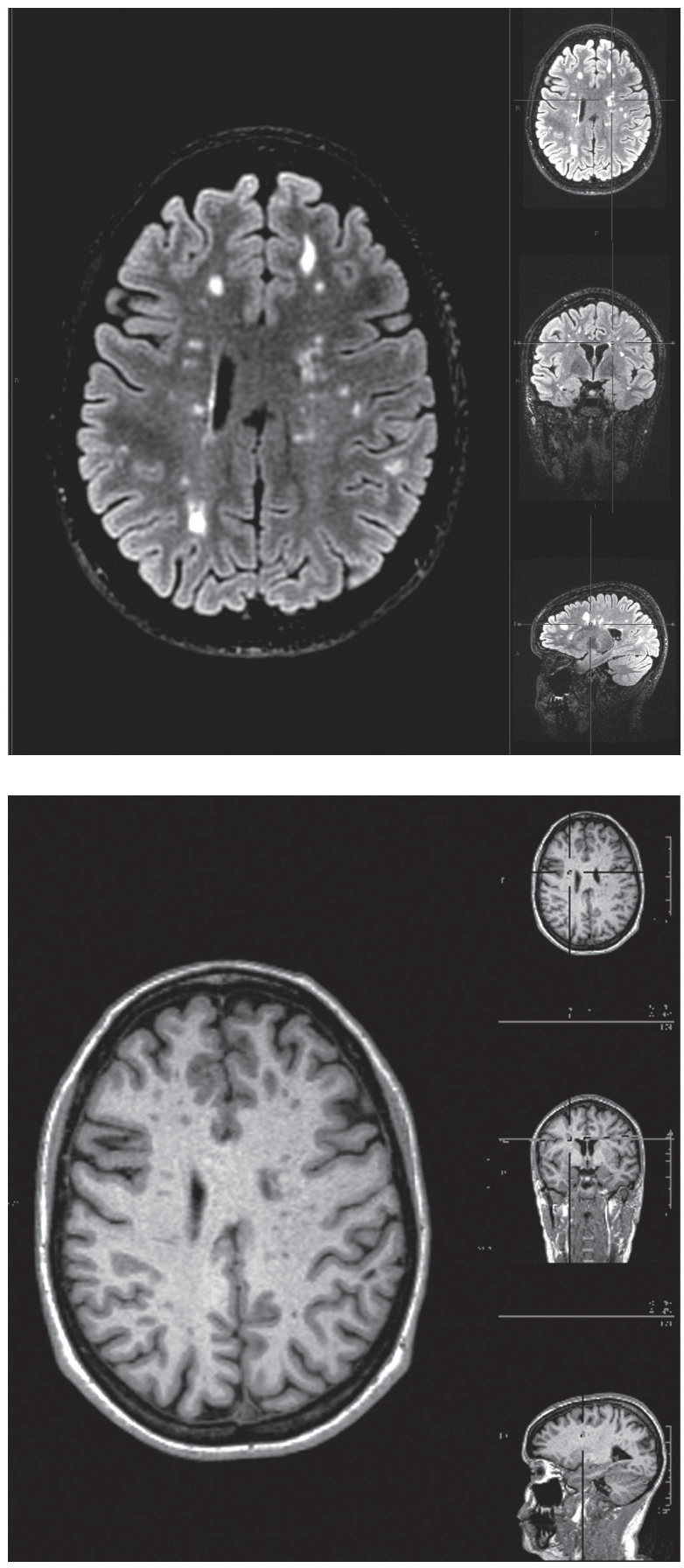

Figura 1. En las reconstrucciones MPR de una secuencia volumétrica FLAIR (a) se observan múltiples lesiones hiperintensas en la sustancia blanca con patrón desmielinizante periventricular, con compromiso de la unión callososeptal, observando la típica morfología de los dedos de Dawson. Se incluye corte similar MPRAGE (b). 


\section{Neurorradiología cuantitativa}

El desarrollo de nuevas secuencias en resonancia, nuevas bobinas, mayor poder en el campo de los magnetos (alto campo) han permitido obtener imágenes con una mayor sensibilidad en relación a los cambios neuropatológicos y una mayor resolución anatómica.

Además, el desarrollo de nuevos softwares tales como FSL (www.fmrib.ox.ac.uk/fsl/(9) y SPM (www.fil. ion.ucl.ac.uk/spm/(10)), permite el análisis e interpretación cuantitativa de los datos obtenidos. Así surge la neurorradiología cuantitativa que cambia la visión que se tenía de la enfermedad, intentando cambiar el manejo de la EM, ya que permite identificar pequeñas variaciones cuantitativas antes indetectables y, por lo tanto, mejora nuestra precisión para evaluar la respuesta individual a un tratamiento determinado.

Las mediciones de la carga lesional y de la actividad inflamatoria de la enfermedad, a través de técnicas cuantitativas de RM se utilizan cada vez con mayor frecuencia, tanto en clínica como en investigación. Estas técnicas requieren un flujo de análisis que permitan asegurar la eficiencia, reproducibilidad y control de calidad de imágenes por resonancia magnética de pacientes con EM, lo que requiere de un procesamiento avanzado de imágenes. Debido a los avances en informática con la implementación de sistemas PACS (Picture Archiving and Communication Systems) se tienen a disposición bases de datos que permiten realizar análisis dirigidos a una patología en particular.

Los softwares para determinar el volumen cerebral utilizan técnicas de segmentación de imágenes, corregistro y análisis de series en el tiempo(11).

Ya que el análisis cuantitativo de las imágenes de $\mathrm{RM}$ se basa en intensidades relativas, como primer paso en el proceso se debe corregir la inhomogeneidad del campo (muy importante en equipos de alto campo, mayor de 3T, y con el uso de bobinas phased-array), luego se hace el registro y segmentación.

En el registro las imágenes realizadas en diferentes equipos se sobreponen en las mismas localizaciones anatómicas $^{(12)}$. El tiempo entre los estudios puede ser de días o años, lo que permite el seguimiento comparativo a largo plazo. Los estudios se pueden realizar con la misma ponderación, por ejemplo T1 para evaluar la atrofia ${ }^{(13)} \mathrm{o}$ ponderación diferente, incluso utilizando diferentes modalidades de imágenes, por ejemplo RM v/s PET.

\section{Medición de la Carga Lesional}

Un importante uso de la segmentación en el estudio de la EM, es determinar la cantidad y volumen de las lesiones hiperintensas en T2, lo cual se puede cuantificar en milímetros cúbicos para comparar con estudios posteriores. Los cambios en el volumen de las lesiones se utilizan en los ensayos clínicos de nuevos tratamientos para la $\mathrm{EM}^{(14,15)}$.

La segmentación de lesiones de la EM se puede hacer manualmente corte por corte por un operador, donde es posible lograr una reproducibilidad razonable, pero es lento y requiere gran uso de mano de obra. Además, se producen errores intraoperador (mismo operador), o interoperador (diferentes operadores).

Múltiples trabajos han abordado el problema de reducir la cantidad de tiempo de operador y mejorar la reproducibilidad de la medición de los volúmenes de las lesiones de EM, utilizando métodos semi o totalmente automatizados ${ }^{(16-18)}$, los que incluyen técnicas de detección de bordes, seguimiento de contornos, redes neuronales, compresión fractal de imágenes, etc.

Es probable que el enfoque más prometedor se base en segmentación usando múltiples parámetros, donde la información de un número de secuencias (por ejemplo T1, DP y FLAIR) se combinan para segmentar las lesiones basadas en la posición de los pixeles en un espacio $\mathrm{N}$-dimensiones, según intensidad.

La clasificación semi-automática de lesiones puede dar una adecuada sensibilidad con una buena reproducibilidad del método en la detección de lesiones de EM, junto a la eliminación de la variabilidad y de la subjetividad (variabilidad intraoperador, interoperador) y la reducción en el costo asociado al proceso manual. Pero todavía es necesario el operador para identificar manualmente algunas lesiones de EM.

La mayoría de los métodos de identificación y segmentación automáticos como semiautomáticos de lesiones se basan en un rango de intensidades de los diferentes tejidos del encéfalo. Sin embargo, grandes variaciones de intensidad pueden violar estos supuestos y afectar negativamente el éxito del proceso. Estas variaciones se derivan de los diferentes fabricantes y modelos de resonadores, las diferencias en los protocolos de adquisición (espesor de corte, etc.), también por ser realizados en diferentes fases de la enfermedad o la presencia de la patología concomitante que puede afectar el comportamiento de la intensidad de los tejidos de manera significativa $^{(19,20)}$. Debido a lo anterior, algunos softwares como TOADS-CRUISE, además de utilizar los niveles de gris para detectar las lesiones de EM, utilizan atlas anatómicos para eliminar falsos positivos ${ }^{(21)}$.

\section{Atrofia cerebral}

La atrofia cerebral es una característica normal del envejecimiento, con pérdida progresiva del tejido encefálico a partir de la edad adulta temprana. Para evaluar la atrofia cerebral, hay que tener en consideración que el volumen del cerebro es altamente variable entre los individuos.

Se cree que la pérdida de tejido neuronal es un indicador de largo plazo de daño irreversible del tejido. Esto es algo complejo cuando existe el fenómeno de 
"'pseudoatrofia global", por el efecto antinflamatorio de algunos tratamientos de EM, donde se produce una rápida pérdida inicial de volumen cerebral debido a una reducción en el exceso de líquido en el cerebro, fenómeno que debe ser considerado al momento de la evaluación volumétrica(22).

La atrofia cerebral se puede medir de forma transversal o longitudinal. La transversal es estimar el volumen total de tejido cerebral. Debido a que el volumen del cerebro es variable, los resultados no son fácilmente comparables, excepto en los estudios de grandes poblaciones. Una manera de estandarizar las medidas, es proporcionar un "normalizado" del volumen del cerebro. Hay una forma de hacer esto: dividir el volumen del parénquima cerebral por el volumen craneal, ya que el volumen craneal permanece fijo durante la vida adulta ${ }^{(23)}$.

En los estudios longitudinales es posible medir la reducción de volumen del cerebro normalizado con respecto al estudio basal. Sin embargo, los registros sofisticados de los estudios realizados en diferentes puntos de tiempo, hacen posible no sólo evaluar la variación bruta de volumen de tejido, sino que también visualizar la ubicación anatómica de la pérdida de tejido(24).

Estudios patológicos y de imágenes sugieren que el desarrollo de deterioro neurológico permanente en la EM, se asocia a la atrofia progresiva cerebral y de la médula espinal.

\section{Material y métodos}

Con resonadores Siemens Avanto y General Electric (GE) Optima 450 de 1.5T, se adquirieron secuencias volumétricas T1 (Secuencia MPRAGE en equipo Siemens con parámetros: TR 2400 ms, TE: 3.74 ms, Ti: 1000 ms, FOV: 250 x 250. Secuencia FSPGR (BRAVO) en equipo GE con parámetros TR: $9.7 \mathrm{~ms}$, TE: $3.7 \mathrm{~ms}$, Ti: $600 \mathrm{~ms}$, FOV: 256 x 256. Secuencia FLAIR en equipo Siemens con parámetros TR: 7000 ms, TE: 430 ms, Ti: 2200 ms, FOV: 256 x 256. Secuencia FLAIR en equipo GE con parámetros TR: 6000 ms, TE: 146.7 ms, Ti: 1812 ms, FOV: 256 x 256) a 50 pacientes derivados por sus médicos tratantes para control por esclerosis múltiple en forma rutinaria, o por la aparición de un brote clínico. De éstos se seleccionaron 11 (9 mujeres y 2 hombres) porque tenían al menos un control con resonancia magnética en el tiempo (promedio: 12.6 meses), cuyas edades fluctuaron entre los 23 y 54 años (promedio: 38 años). El seguimiento con resonancia se realizó ente los 5 y 15 meses (promedio: 12.6 meses). Se realizó el análisis volumétrico con software Freesurfer v.4.5.0 (Freesurfer, http://surfer. nmr.mgh-harvard.edu; ejemplo de segmentación) y con software MIPAV con el plugin TOADS-CRUISE (http://www.nitrc.org/projects/toads-cruise/) para recuento lesional, el que permite utilizar secuen- cias T1, FLAIR, T2 y PD, pero se obtienen mejores resultados utilizando secuencias T1 y FLAIR. Se comparó con nuestra base de datos volumétricos normales según rango etario.

Con el fin de comparar las imágenes de los distintos pacientes es necesario transformarlas en un espacio anatómico estándar ${ }^{(12)}$. En nuestros casos nos basamos en el atlas cerebral MNI, del Instituto Neurológico de Montreal, el que está compuesto por el registro de los estudios de una amplia muestra de sujetos controles, de los cuales se obtiene un cerebro promedio, el cual fue corregistrado de forma aproximada al atlas de Talairach y Tournoux ${ }^{(26)}$.

\section{Resultados}

En figura 2 se muestra un corte coronal de la segmentación de un paciente con EM, obtenida con Freesurfer. En figura 3 se muestran cortes axiales de la segmentación de lesiones de EM del mismo paciente anterior, obtenidas con TOADS-CRUISE. En figura 4 se muestra la reconstrucción 3D de las lesiones de EM segmentadas previamente con TOADS-CRUISE y reconstruidas con 3D Slicer. En figura 5 se comparan dos reconstrucciones $3 \mathrm{D}$ de cargas lesionales de un mismo paciente en un año de evolución. Los círculos rojos indican zonas con aumento de la carga lesional.

En figura 6 y Tabla I se muestra el formato que nos entrega en nuestros pacientes el estudio volumétrico individual con Freesurfer. En este caso visualizamos una leve disminución de volumen cerebral difuso en el plazo de un año.

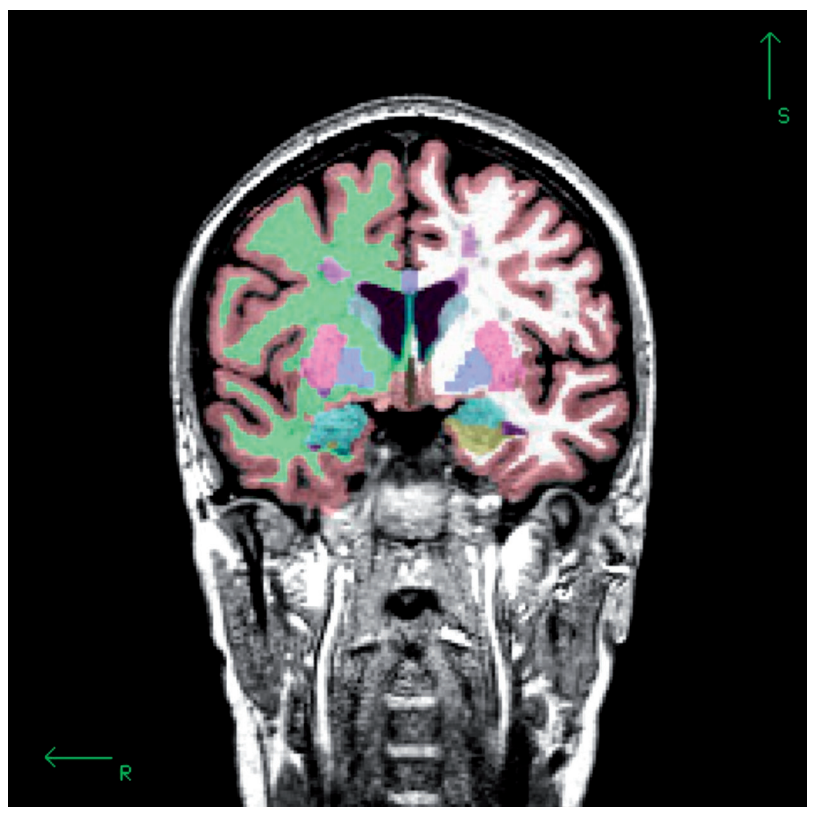

Figura 2. Imagen de segmentación del encéfalo diferenciando la sustancia blanca y gris, imagen en el plano coronal realizada con Freesurfer. 

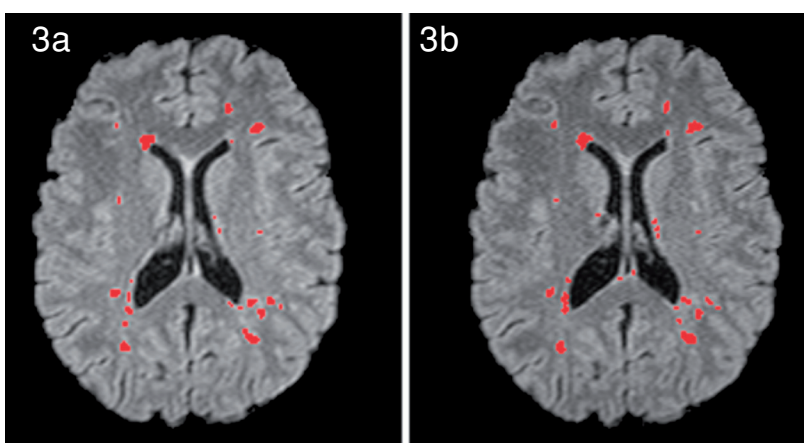

Figura 3. Cortes axiales de carga lesional segmentada con MIPAV en un mismo paciente entre la resonancia del (a) 04-10-2010 y del (b) 14-10-2011. (volumetría cerebral en tabla 1)
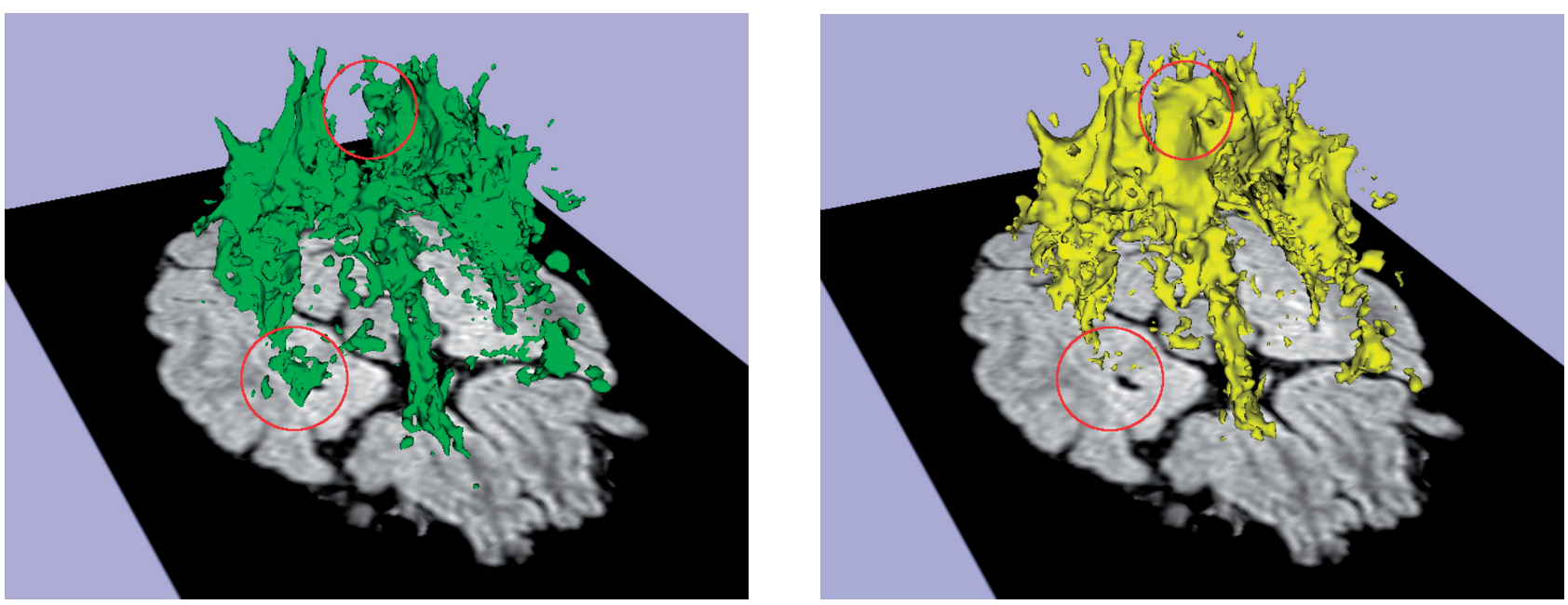

Figura 5. Reconstrucciones $3 D$ comparativas de cargas lesionales de un mismo paciente en un año de evolución. Los círculos rojos indican zonas con aumento de la carga lesional.
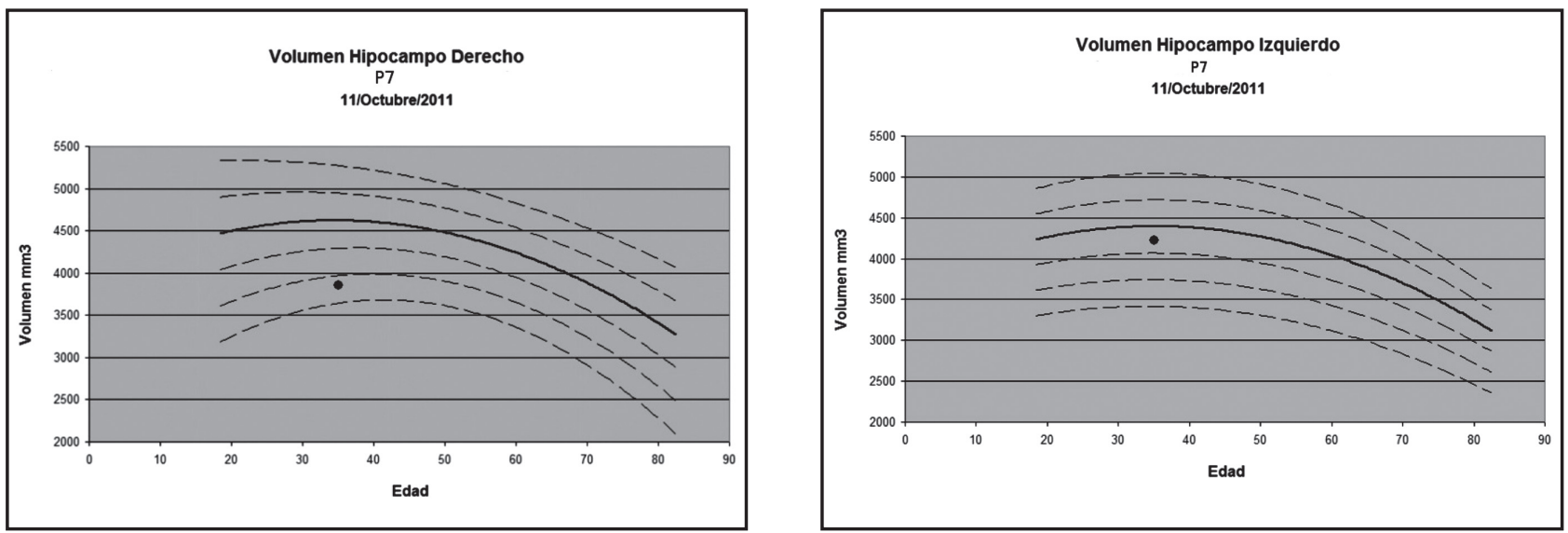

Figura 6. Gráfico de la variación del volumen hipocampal derecho e izquierdo según la edad. Se especifica (ver círculos) el volumen hipocampal de paciente P7. 
Tabla I. Seguimiento volumétrico de un paciente con EM obtenidos con Freesurfer con un año de diferencia en una paciente con esclerosis múltiple RR, donde se detalla el análisis volumétrico de las estructuras encefálicas, demostrando una disminución del volumen cerebral de un $5 \%$.

Volúmenes absolutos

\begin{tabular}{|c|c|c|c|}
\hline & $\begin{array}{l}14 / 10 / 2010 \\
\text { (cc) }\end{array}$ & $\begin{array}{l}4 / 10 / 2011 \\
\quad(c c)\end{array}$ & $\begin{array}{l}\text { Valores normales } \\
\text { (cc) }\end{array}$ \\
\hline Volumen intracraneano total: & 1536 & 1536 & normal: 1485,4 DS: 123,4 \\
\hline Volumen cerebral: & 1001 & 954 & normal: 984,1 DS: 88,0 \\
\hline Volumen sustancia blanca hemisferio izquierdo: & 227,8 & 216,4 & normal: 217,2 DS: 19,4 \\
\hline Volumen corteza cerebral hemisferio izquierdo: & 239,4 & 227,5 & normal: 243,8 DS: 23,6 \\
\hline Hipocampo izquierdo: & 4,3 & 4,2 & normal: 4,2 cc DS: 0,4 \\
\hline Amígdala izquierda: & 1,5 & 1,5 & normal: 1,7 DS: 0,2 \\
\hline Ventrículo lateral izquierdo: & 8,4 & 9,0 & normal: 7,1 DS: 2,6 \\
\hline Volumen sustancia blanca hemisferio derecho: & 245,1 & 223,0 & normal: 219,7 DS: 19,1 \\
\hline Volumen corteza cerebral hemisferio derecho: & 229,2 & 228,4 & normal: 244,6 DS: 24,6 \\
\hline Hipocampo derecho: & 4,0 & 3,9 & normal: $4,5 \mathrm{cc}$ DS: 0,5 \\
\hline Amígdala derecha: & 1,6 & 1,5 & normal: $1,7 \mathrm{DS}: 0,2$ \\
\hline Ventrículo lateral derecho: & 10,8 & 11,3 & normal: 6,8 DS: 2,6 \\
\hline 3er Ventrículo: & 0,8 & 0,8 & normal: 0,9 DS: 0,1 \\
\hline \multicolumn{4}{|l|}{ Volúmenes relativos } \\
\hline & $14 / 10 / 2010$ & $4 / 10 / 2011$ & Valores normales \\
\hline Volumen sustancia blanca hemisferio izquierdo: & 0,1483 & 0,1409 & normal: 0,1463 DS: 0,0071 \\
\hline Volumen corteza cerebral hemisferio izquierdo: & 0,1559 & 0,1481 & normal: 0,1642 DS: 0,0108 \\
\hline Hipocampo izquierdo: & 0,0028 & 0,0027 & normal: 0,0028 DS: 0,0002 \\
\hline Amígdala izquierda: & 0,0010 & 0,0010 & normal: 0,0012 DS: 0,0001 \\
\hline Ventrículo lateral izquierdo: & 0,0054 & 0,0058 & normal: 0,0048 DS: 0,0015 \\
\hline Volumen sustancia blanca hemisferio derecho: & 0,1596 & 0,1452 & normal: 0,1480 DS: 0,0080 \\
\hline Volumen corteza cerebral hemisferio derecho: & 0,1492 & 0,1487 & normal: 0,1647 DS: 0,0115 \\
\hline Hipocampo derecho: & 0,0026 & 0,0025 & normal: 0,0030 DS: 0,0002 \\
\hline Amígdala derecha: & 0,0010 & 0,0010 & normal: 0,0011 DS: 0,0001 \\
\hline Ventrículo lateral derecho: & 0,0071 & 0,0074 & normal: 0,0046 DS: 0,0017 \\
\hline 3er Ventrículo: & 0,0005 & 0,0006 & normal: 0,0006 DS: 0,0001 \\
\hline
\end{tabular}

El $36 \%$ de los pacientes estudiados presentaron disminución de volumen cerebral difuso (Figura 7), pero debemos diferenciar si se afecta más la sustancia blanca o la corteza cerebral. La disminución de volumen de la sustancia gris cortical $(72 \%$ de los 11 pacientes) afectó un mayor número de pacientes que la disminución de volumen de la sustancia blanca (39\%, Figura 8).
El $54 \%$ de los pacientes estudiados presentó una disminución de volumen de uno o ambos hipocampos (Figura 9).

El estudio con MIPAV nos permite comparar la carga lesional en un mismo paciente, lo cual se puede visualizar de forma tridimensional. El $63 \%$ de los pacientes estudiados presentó aumento de la carga lesional (Figura 10). 


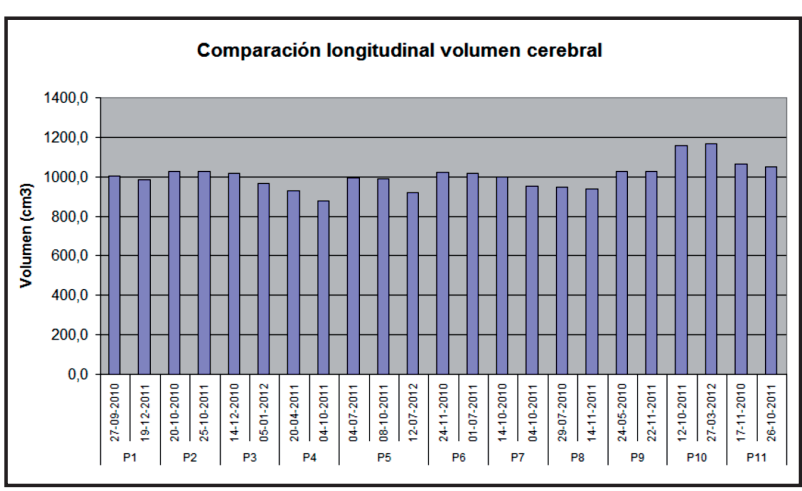

Figura 7. Gráfico de barras en que se compara el volumen cerebral total en el tiempo, de los 11 pacientes con esclerosis múltiple. El $36 \%$ de los pacientes estudiados, presentaron disminución de volumen cerebral difuso.

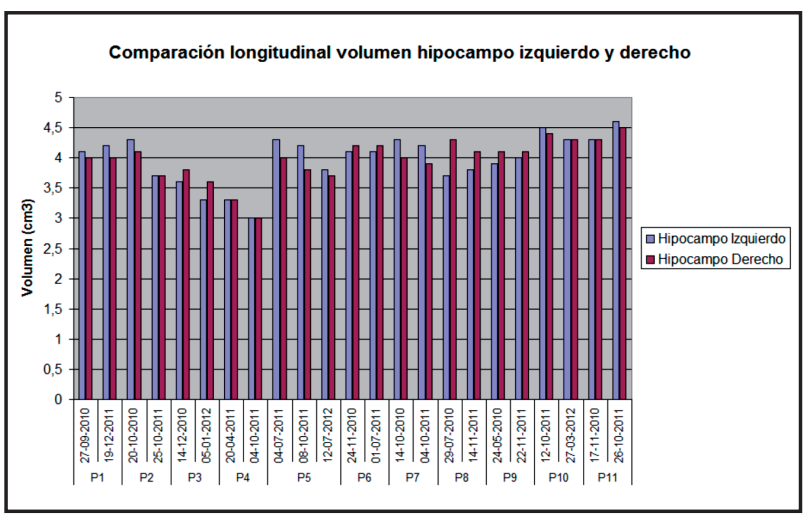

Figura 9. Gráfico de barras donde se compara la evolución en el tiempo de los volúmenes hipocampales de los 11 pacientes con esclerosis múltiple. El $54 \%$ de los pacientes estudiados presentó disminución de volumen de uno o ambos hipocampos.

\section{Discusión}

La atrofia se ha sugerido como un marcador potencial de progresión de la enfermedad. El registro de resonancias seriadas ofrece una alternativa útil para evaluar la atrofia cerebral con esta técnica, ya que las mediciones son sensibles y reproducibles, lo que permite detectar la atrofia progresiva dentro de plazos tan cortos como, por ejemplo, un año de evolución de una enfermedad y puede tener potencial como un marcador de la progresión de la enfermedad en el seguimiento de los ensayos terapéuticos. En el estudio de Fox et al (2006) ${ }^{(26)}$, los volúmenes cerebrales basales en el grupo de EM fueron menores $\mathrm{v} / \mathrm{s}$ los controles, la tasa de atrofia cerebral en el grupo de EM fue $0,8 \%$ por año, más del doble que la de los controles $(0,3 \%)$. La tasa de crecimiento ventricular fue cinco veces mayor que los controles ${ }^{(28)}$, lo cual nosotros visualizamos en el $36 \%$ de los casos.

El deterioro cognitivo es un importante evento

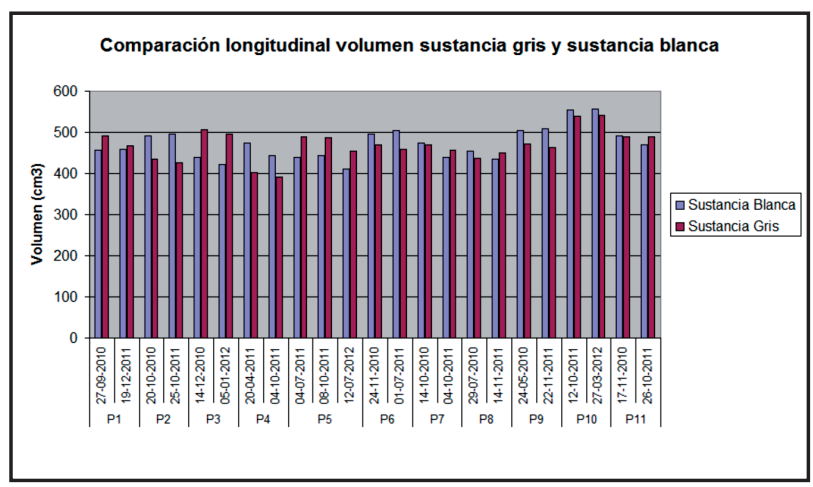

Figura 8. Gráfico de barras en el que se compara el volumen de la sustancia gris y de la sustancia blanca en el tiempo de los 11 pacientes en control por esclerosis múltiple. El $72 \%$ de los pacientes presentó disminución de volumen cortical y un $39 \%$ disminución de volumen de sustancia blanca.

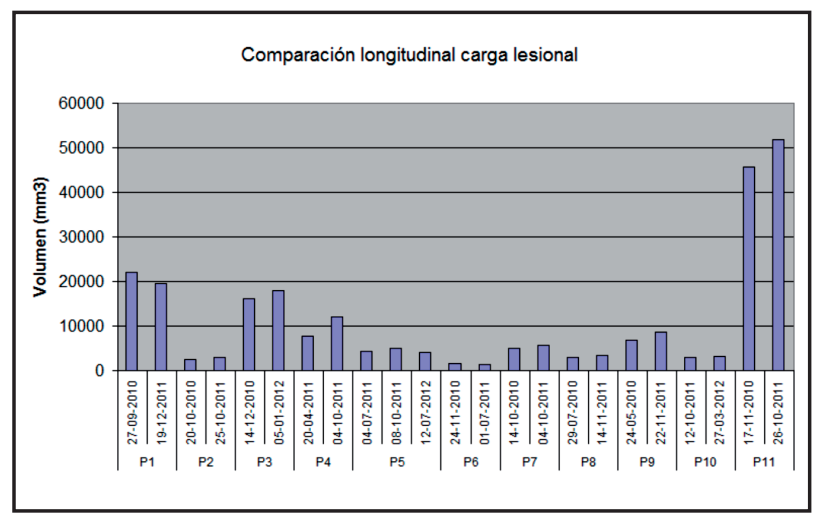

Figura 10. Gráfico de barras en que se compara la evolución en el tiempo de la carga lesional, en los 11 pacientes con esclerosis múltiple. El 63\% de los pacientes estudiados presentó aumento de la carga lesional

clínico en la evolución de la EM, dependiendo de la fase y tipo de la enfermedad, entre un $45-65 \%$ desarrollará un grado variable de disfunción cognitiva. Los estudios patológicos y por RM han fallado en demostrar una estricta relación entre el deterioro cognitivo y la patología en la sustancia blanca subcortical. La correlación es más pobre aun cuando se considera la atrofia global (sustancia blanca más corteza).

En la actualidad está ampliamente aceptado el compromiso de la sustancia gris en la EM. Lesiones desmielinizantes son comunes en la corteza y en las regiones subcorticales ${ }^{(28)}$. Focos de desmielinización subpial también se pueden extender a la corteza. Estos son probablemente relacionados a los nódulos de infiltración de células $B$ en las meninges suprayacentes ${ }^{(29)}$ y puede reflejar la acción de anticuerpos antimielina. La atrofia cortical y subcortical de la sustancia gris es progresiva durante la evolución de la enfermedad, la cual puede atribuirse a una distrofia neural, pero en los núcleos subcorticales, como el 
tálamo la atrofia se asocia con pérdida neuronal(30,31). Se ha observado una significativa correlación negativa entre el espesor cortical promedio y el volumen total de las lesiones de sustancia blanca, lo cual da sostén al uso de medición de carga lesional y espesor cortical promedio(32,33). Nosotros cuantificamos que el $63 \%$ presentó aumento de la carga lesional.

Para nuestro análisis utilizamos el software Freesurfer v.4.5.0 (34-36) para analizar el volumen cortical así como el espesor cortical (este dato será objeto de publicaciones posteriores). Este software por defecto nos entrega los valores de volumen cortical total (Figura 2), lo que es incluido en la evaluación de pacientes con EM, especialmente si tienen algún grado de déficit cognitivo.

El rol primario de la patología cortical en EM por lesiones inflamatorias focales y atrofia cortical, ha sido subestimado. Éstas determinan déficit cognitivos focales (deterioro de memoria, déficit de atención, procesamiento del lenguaje) y atrofia global, lo que está determinado por diferentes estudios ${ }^{(37-40)}$.

Nuestros hallazgos, los cuales son semejantes a lo encontrado en la experiencia publicada en otros centros, nos hacen comprender que los avances técnicos en los equipos de resonancia magnética, así como las nuevas técnicas de procesamiento de imágenes, nos permiten obtener parámetros cuantitativos como volumen cerebral global y de las diferentes estructuras encefálicas, especialmente la sustancia blanca y el cortical, así como la carga lesional, lo cual nos permite evaluar cuantitativamente la evolución temporal de los pacientes con esclerosis múltiple. Esto marca una gran diferencia respecto a los protocolos de resonancia estándar utilizados en la rutina clínica de nuestro país. Esto nos ha permitido determinar los cambios morfovolumétricos durante los controles anuales de resonancia magnética en aquellos pacientes sin brotes aparentes de su enfermedad, determinando con sorpresa que a pesar del o de los tratamiento utilizados para detener la progresión de la enfermedad, se observa la aparición de nuevas lesiones con aumento de la carga lesional y disminución del volumen cerebral.

La utilización de las técnicas cuantitativas para la valoración de volumen cerebral y recuento lesional en pacientes con esclerosis múltiple no han sido públicas en nuestro país hasta la fecha.

Dentro de las limitaciones de esta técnica debemos mencionar la dificultad de la segmentación de las lesiones en la fosa posterior, debido a la menor diferencia de señal entre la sustancia blanca y gris en esta región y la dificultad de la técnica para diferenciar entre corteza cerebelosa y lesiones, por lo tanto la exactitud de la técnica es deficiente para el conteo de lesiones en la fosa posterior, pero esta limitación no altera la comparación en un mismo sujeto, ya que la omisión de lesiones en esta región es constante en los diferentes estudios de un paciente y no condiciona una variación significativa de la carga lesional, lo cual si es determinado por las lesiones supratentoriales las que son de mayor volumen y número y que sí son bien definidas por esta técnica. Sería interesante tener la correlación de nuestros hallazgos con los estudios neuropsicológicos de los pacientes estudiados así como la exacta correlación del tratamiento que cada uno de ellos utilizaba y su evolución con ellos, lo cual será objeto de estudios posteriores.

En resumen, la volumetría encefálica junto al conteo automatizado de lesiones en pacientes con esclerosis múltiple permite hacer el seguimiento longitudinal de los pacientes, comparando estudios de diferentes fechas con múltiples parámetros en forma reproducible y confiable. Si esto se realiza en forma precoz en el curso de la enfermedad podemos pretender mejorar el manejo de esta patología, con la esperanza que las nuevas terapias en desarrollo logren detener el curso de la enfermedad y lograr mejorías sustanciales del pronóstico a largo plazo, evitando la producción de nuevas lesiones y la consecuente atrofia cerebral, que son los responsables de los daños neurológicos irreversibles que visualizamos en la actualidad.

\section{Bibliografía}

1. Confavreux $C$, Vukusic $S$. Natural history of multiple sclerosis: a unifying concept. Brain 2006; 129: 606616.

2. Frohman EM, Goodin DS, Calabresi PA, Corboy JR, Coyle PK, Filippi M. The utility of MRI in suspected MS Report of the Therapeutics and Technology Assessment Subcommittee of the American Academy of Neurology. Neurology 2003; 61; 602-611.

3. Lucchinetti C, Bruck W, Parisi J, Scheithauer B, Rodríguez M, Lassmann $\mathrm{H}$. Heterogeneity of multiple sclerosis lesions: implications for the pathogenesis of demyelination. Ann Neurol 2000; 47: 707-717.

4. McDonald WI, Compston G, Edan G, Goodkin D, Hartung HP, Lublin FD, et al. Recommended diagnostic criteria for multiple sclerosis: guidelines from the International Panel on the Diagnosis of Multiple Sclerosis. Ann Neurol 2001; 50: 121-127.

5. Lee MA, Smith S, Palace J, Narayanan S, Silver N, Minicucci L, et al. Spatial mapping of T2 and gadoliniumenhancing $\mathrm{T} 1$ lesion volumes in multiple sclerosis: evidence for distinct mechanisms of lesion genesis? Brain 1999; 122: 1261-1270.

6. Lycklama à Nijeholt G, Barkhof F. Differences between subgroups of MS: MRI findings and correlation with histopathology. J Neurol Sci 2003; 206: 173-174.

7. Smith ME, Stone LA, Albert PS, Frank JA, Martin $\mathrm{R}$, Armstrong M, Maloni $\mathrm{H}$, et al. Clinical worsening in multiple sclerosis is associated with increased frequency and area of gadopentetate dimeglumineenhancing magnetic resonance imaging lesions. Ann Neurol 1993; 33: 480-489.

8. Arnold DL, Matthews PM. MRI in the diagnosis and management of multiple sclerosis. Neurology 2002; 58: S23-S31. 
9. Smith SM, Jenkinson M, Woolrich MW, Beckmann $\mathrm{CF}$, Behrens TEJ, Johansen-Berg $\mathrm{H}$, et al. Advances in functional and structural MR image analysis and implementation as FSL. Neurolmage 2004; 23(S1): 208-219.

10. Friston K. Introduction: experimental design and statistical parametric mapping. In Frackowiak et al. (Eds.) Human brain function, 2nd Edition, 2003.

11. Liu L, Meier D, Polgar-Turcsanyi M, et al. Multiple sclerosis medical image analysis and information management. J Neuroimaging 2005; 15(4 Suppl): 103S-117S.

12. Hill DLG, Batchelor PG, Holden M, Hawkes DJ. Medical image registration. Phys Med Biol 2001; 46: R1-R45.

13. Fox NC, Freeborough PA. Brain atrophy progression measured from registered serial MRI: Validation and application to Alzheimer's disease. J Magn Reson Imaging. 1997; 7: 1069-1075.

14. Filippi M, Horsfield MA, Adèr HJ, Barkhof F, Bruzzi $P$, Evans $A$, et al. Guidelines for using quantitative measures of brain magnetic resonance imaging abnormalities in monitoring the treatment of multiple sclerosis. Ann Neurol 1998; 43: 499-506.

15. Tomassini $V$, Paolillo A, Russo P, Giugni E, Prosperini L, Gasperini. C. Predictors of long-term clinical response to interferon beta therapy in relapsing multiple sclerosis. J Neurol 2006; 253: 287-293.

16. Grimaud J, Lai M, Thorpe J, Adeleine P, Wang L, Barker GJ, et al. Quantification of MRI lesion load in multiple sclerosis: a comparison of three computer-assisted techniques. Magn Reson Imaging 1996; 14: 495-505.

17. Raff $U$, Vargas PF, Rojas GM, Scherzinger AL, Simon $\mathrm{JH}$. Quantitation of T2 Lesion Load in Multiple Sclerosis with Magnetic Resonance Imaging: A Pilot Study of a Probabilistic Neural Network Approach. Acad Radiol 1997; 4: 431-437.

18. Raff U, Rojas GM, Hutchinson M, Simon JH. Quantitation of T2 Lesion Load in Patients with Multple Sclerosis: A Novel Semiautomated Segmented Technique. Acad Radiol 2000; 7: 237-247

19. Horsfield MA, Bakshi R, Rovaris M, Rocca MA, Dandamudi VS, Valsasina $P$, et al. Incorporating domain knowledge into the fuzzy connectedness framework: Application to brain lesion volume estimation in multiple sclerosis. IEEE Trans Med Imaging 2007; 26: 16701680.

20. Arnold DL, Arbel T. Medical Image Analysis on Multiple Sclerosis: validation and methodological issues. MICCAI Workshop on. Med. Imag. Analysis on Multiple Sclerosis, 2009; 15-26.

21. Shiee N, Bazin PL, Ozturk A, Reich DS, Calabresi PA, Pham DL. A topology-preserving approach to the segmentation of brain images with multiple sclerosis lesions. Neuroimage 2010; 49: 1524-1535.

22. Fisher E, Rudick RA, Simon JH, Cutter G, Baier M, Lee $\mathrm{JC}$, et al. Eight-year follow-up study of brain atrophy in patients with MS. Neurology 2002; 59: 1412-1420.

23. Smith SM, Zhang YY, Jenkinson M, Chen J, Matthews PM, Federico A, et al. Accurate, robust, and automated longitudinal and cross-sectional brain change analysis. Neuroimage 2002; 17: 479-489.

24. Freeborough PA, Fox NC. Modeling brain deformations in Alzheimer disease by fluid registration of serial 3D MR images. J Comput Assist Tomogr 1998; 22: 838-843.
25. Collins DL, Neelin P, Peters TM, Evans AC. Automatic 3D intersubject registration of MR volumetric data in standardized Talairach space. J Comput Assist Tomogr 1994; 18:192-205.

26. Talairach J, Tournoux P. Co-planar Stereotaxic Atlas of the Human Brain: 3-Dimensional Proportional System: An Approach to Medical Cerebral Imaging. Stuttgart: Thieme Medical Publishers; 1988t. 122 pp.

27. Fox NC, Jenkins R, Leary SM, Stevenson VL, Losseff NA, Crum WR, et al. Progressive cerebral atrophy in MS - a serial study using registered, volumetric MRI. Neurology 2000; 54: 807-812.

28. Wegner C, Esiri MM, Chance SA, Palace J, Matthews PM. Neocortical neuronal, synaptic, and glial loss in multiple sclerosis. Neurology 2006; 67: 960-967.

29. Magliozzi R, Howell O, Vora A, Serafini B, Nicholas $\mathrm{R}$, Puopolo M, et al. Meningeal Bcell follicles in secondary progressive multiple sclerosis associate with early onset of disease and severe cortical pathology. Brain 2007; 130: 1089-1104.

30. Chen JT, Narayanan S, Collins DL, Smith SM, Matthews PM, Arnold DL. Relating neocortical pathology to disability progression in multiple sclerosis using MRI. Neuroimage 2004; 23: 1168-1175.

31 Cifelli A, Arridge M, Jezzard P, Esiri MM, Palace J, Matthews PM. Thalamic neurodegeneration in multiple sclerosis. Ann Neurol 2002; 52: 650-653.

32. Dickerson BC, Fenstermacher E, Salat DH, Wolk DA, Maguire RP, Desikan R, et al. Detection of cortical thickness correlates of cognitive performance: Reliability across MRI scan sessions, scanners, and field strengths. Neuroimage 2008; 39: 10-18.

33. Charil A, Dagher A, Lerch JP, Zijdenbos AP, Worsley $\mathrm{KJ}$, Evans AC. Focal cortical atrophy in multiple sclerosis: Relation to lesion load and disability. Neuroimage 2007; 34: 509-517.

34. Dale AM, Fischl B, Sereno MI. Cortical surface-based analysis. I. Segmentation and surface reconstruction. Neuroimage 1999; 9: 179-194.

35. Fischl B, Sereno MI, Dale AM. Cortical surface-based analysis. II: inflation, flattening, and a surface-based coordinate system. Neuroimage 1999; 9: 195-207.

36. Fischl B, Dale AM. Measuring the thickness of the human cerebral cortex from magnetic resonance images. Proc Natl Acad Sci USA 2000; 97: 11050-11055. Freesurfer (http://surfer.nmr.mgh-harvard.edu/)

37. Ramasamy DP, Benedict RH, Cox JL, Fritz D, Abdelrahman N, Hussein S, et al. Extent of cerebellum, subcortical and cortical atrophy in patients with MS. A case-control study. J Neurol Sci 2009; 282: 47-54.

38. Calabrese M, Rinaldi F, Grossi P, Gallo P. Cortical pathology and cognitive impairment in multiple sclerosis. Expert Rev Neurother 2011; 11: 425-432.

39. Grassiot B, Desgranges B, Eustache F, Defer G. Quantification and clinical relevance of brain atrophy in multiple sclerosis: a review. J Neurol 2009; 256: 1397-1412.

40. Matthews PM. Brain Imaging of Multiple Sclerosis: the Next 10 Years. Neuroimaging Clin N Am 2009; 19: 101-112.

41. Shiee N, Bazin PL, Zackowski KM, Farrell SK, Harrison DM. Revisiting Brain Atrophy and Its Relationship to Disability in Multiple Sclerosis. PLoS One. 2012; 7(5): e37049. 